Article

\title{
Anthropometric Status among 6-9-Year-Old School Children in Rural Areas in Hai Phong City, Vietnam
}

\author{
Ngan T.D. Hoang $\left.{ }^{1} \mathbb{(}\right)$, Liliana Orellana $\left.{ }^{2} \mathbb{(}\right)$, Tuyen D. Le ${ }^{3}$, Rosalind S. Gibson ${ }^{4}$, \\ Anthony F. Worsley ${ }^{1}$, Andrew J. Sinclair ${ }^{5}$ and Ewa A. Szymlek-Gay ${ }^{1, *(\mathbb{D}}$ \\ 1 Institute for Physical Activity and Nutrition (IPAN), School of Exercise and Nutrition Sciences, \\ Deakin University, Melbourne, VIC 3125, Australia; hoangthiducngan@dinhduong.org.vn (N.T.D.H.); \\ anthony.worsley@deakin.edu.au (A.F.W.) \\ 2 Biostatistics Unit, Faculty of Health, Deakin University, Melbourne, VIC 3125, Australia; \\ 1.orellana@deakin.edu.au \\ 3 National Institute of Nutrition, Hanoi 10000, Vietnam; ledanhtuyen@dinhduong.org.vn \\ 4 Department of Human Nutrition, University of Otago, PO Box 56, \\ Dunedin 9054, New Zealand; rosalind.gibson@otago.ac.nz \\ 5 Faculty of Health, Deakin University, Geelong, VIC 3216, Australia; andrew.sinclair@deakin.edu.au \\ * Correspondence: ewa.szymlekgay@deakin.edu.au; Tel.: +61-3-9244-5404; Fax: +61-3-9244-6017
}

Received: 17 August 2018; Accepted: 26 September 2018; Published: 4 October 2018

\begin{abstract}
A double burden of malnutrition in Vietnamese children has emerged as a key challenge: childhood undernutrition remains a public health concern while childhood overweight/obesity has gradually increased. This study aimed to (1) estimate the prevalence of undernutrition and overnutrition among 6-9-year-old primary school children in rural areas of Vietnam, and (2) identify sociodemographic factors associated with undernutrition and overnutrition in this population. A cross-sectional survey was conducted in October 2016 in 2334 children from eight primary schools in rural areas in Hai Phong City, Vietnam. Anthropometric and demographic data were collected. The prevalence of underweight, stunting, wasting, and anthropometric failure was $8.0 \%$, $5.1 \%, 5.3 \%$, and $11.9 \%$, respectively. Up to $22.1 \%$ of children were affected by overweight/obesity, and $31.0 \%$ by abdominal overweight/obesity. Low maternal education was associated with higher odds of underweight and anthropometric failure, whereas overweight/obesity or abdominal overweight/obesity were more likely in boys and children of mothers with a high education level. This study provides evidence for a double burden of diseases among primary school children in rural areas in Hai Phong City. Future interventions for the prevention and control of childhood undernutrition and overweight/obesity should take into account child sex and maternal education level.
\end{abstract}

Keywords: Vietnam; malnutrition; undernutrition; overweight and obesity; abdominal obesity; double burden of diseases; school children

\section{Introduction}

Child malnutrition, which encompasses both undernutrition as well as overnutrition, continues to be a global challenge [1,2]. Recent estimates indicate that up to $25 \%$ of preschool children in low- and middle-income countries are at risk of stunting, $15 \%$ of underweight, and $8 \%$ of wasting [3]. This high prevalence of undernutrition persists into later childhood with up to a third of school children reported as underweight or stunted [4-7]. Similarly, while overweight and obesity are a growing problem in preschool children with $6.0 \%$ of the world's children affected [8], low-resource regions such as South-eastern Asia, are reported to have the highest prevalence of overweight affecting $10.7 \%$ of 
under-five-year-olds [8]. The prevalence of overweight and obesity is even greater among school children in South-eastern Asia with more than $30 \%$ of children affected in some settings [9-11].

Childhood undernutrition also remains a key challenge for Vietnam. Although the prevalence of underweight in children under 5 years of age has decreased considerably from 39.0\% in 1999 to $14.1 \%$ in 2015, the prevalence of stunting still remains high (24.6\% in 2015) [7] and is of public health concern [12]. The increased prevalence of overweight and obesity among Vietnamese children is also concerning. While few children were obese in the late 20th century, by 2005 approximately $36.8 \%$ of preschool children have been affected [13]. However, anthropometric data in primary school children are limited. The most recent study, which included 2,872 children aged 5-11 years from 6 provinces in Vietnam, found that the prevalence of underweight and stunting was still high in both urban and rural areas (underweight: up to $25 \%$; stunting: up to $17.7 \%$ ), and that overweight/obesity affected up to $33.7 \%$ of children [14]. Prevalence data for undernutrition and overnutrition from school children residing in other provinces are still lacking.

Undernutrition is a public health concern as it is the main cause of child mortality $[15,16]$ due to its role in reducing immune competence and thus increasing susceptibility to infections such as pneumonia, diarrhoea, or measles [16]. In contrast, childhood obesity has been identified as a risk factor for obesity in adulthood which in turn contributes to the development of cardiovascular disease, type 2 diabetes and its associated retinal and renal complications, asthma, infertility, psychiatric disorders, cancer, and other obesity-related disorders [17]. Clearly, this dual burden of diseases needs to be addressed and requires strong effort from the healthcare system [18]. However, to enable Vietnam's limited resources to be allocated to the most vulnerable populations, research needs to identify children at higher risk of undernutrition and overnutrition. Therefore, the aims of this study were 1) to estimate the prevalence of undernutrition (i.e., underweight, stunting, wasting, and aggregate anthropometric failure) and overnutrition (i.e., overweight/obesity and abdominal overweight/obesity) among 6-9-year-old primary school children in rural areas in Hai Phong City, Vietnam, and 2) to identify sociodemographic factors associated with undernutrition and overnutrition in this population.

\section{Materials and Methods}

\subsection{Study Design}

This study used screening data from an intervention trial that investigated the effects of micronutrient supplementation for improving micronutrient status, growth, and health outcomes in underweight and overweight/obese primary school children in Vietnam (registered at www.actr.org.au as ACTRN12616001245482). A school-based cross-sectional survey of 6-9-year-old children $(n=2334)$ was conducted in eight primary schools in rural areas in Hai Phong City, Vietnam, in October 2016. Anthropometric measurements and self-reported sociodemographic data were collected. Ethical approval for the study was obtained from the Ethics Committee of the National Institute of Nutrition in Vietnam (610/VDD-QLKH; dated 30/9/2016) and the Deakin University Human Research Ethics Committee in Australia (2016-181). Written informed consent was obtained from the child's primary caregiver and verbal consent was obtained from each participating child.

\subsection{Schools Selection and Recruitment}

Hai Phong City is administratively divided into eight rural districts and seven urban districts; this research was conducted in rural districts only. Schools located in rural areas of Hai Phong City were selected using a multistage sampling approach. In the first stage, two districts (Thuy Nguyen and An Lao) were randomly selected from the eight rural districts in Hai Phong City. In the second stage, schools were selected from the list of all primary schools in each district $(n=38$ in Thuy Nguyen and $n=19$ in An Lao). Schools with $<300$ students were excluded. Selected schools were invited to take part through written invitations followed by telephone calls, emails, and/or visits to explain 
the purpose of this study, confirm participation, and obtain written informed consent from school principals. When a school declined to take part in this study, the next school on the list was invited until four were enrolled in each district.

\subsection{Participants and Recruitment}

Once a school agreed to participate, all children attending grades 1 to 3 (i.e., children aged 6-9 years) were invited to take part. Child's date of birth was obtained from the school registration form in order to confirm the child's age.

Children were excluded if they were older than 9 years, had anthropometric abnormalities (e.g., severe scoliosis which would not allow for correct determination of height), or had an intellectual impairment that would prevent them from understanding the aims of the study.

\subsection{Anthropometric Measures}

Children were weighed in light clothing and without shoes with calibrated electronic body scales (TANITA BC-543, TANITA Corporation, Tokyo, Japan). Each child was weighed twice to the nearest $0.1 \mathrm{~kg}$; if the two measurements differed by more than $0.1 \mathrm{~kg}$, a third measurement was taken.

Height was measured twice to the nearest $0.1 \mathrm{~cm}$ with a SECA stadiometer (SECA 222, SECA $\mathrm{GmbH} \& \mathrm{Co}$. KG, Hamburg, Germany). Children were measured without any hair ornaments, braids or hats, and without shoes or socks. If the duplicate measurements differed by more than $0.1 \mathrm{~cm}$, a third measurement was taken.

Waist circumference (WC) was measured twice horizontally to the nearest $0.1 \mathrm{~cm}$ with a nonstretchable tape (Lufkin W606PM, Apex Tool Group, MD, USA) at a point halfway between the lowest rib and the top of a child's hipbone [19]. If the two measurements differed by more than $0.1 \mathrm{~cm}$, a third measurement was taken.

The final weight, height, and WC were calculated as the mean of the two or three measurements obtained for each child, as appropriate. All anthropometric measurements were taken by trained staff from the National Institute of Nutrition in Vietnam according to standardised procedures [19].

Body Mass Index (BMI) was calculated. Weight-for-age, height-for-age, and BMI-for-age $z$-scores were calculated with the WHO Anthro Plus software version 2.0 [20]. Underweight, stunting, wasting, and overweight/obesity were defined according to the WHO classification [21], i.e., underweight: weight-for-age $z$-score $<-2$; stunting: height-for-age $z$-score $<-2$; wasting: BMI-for-age $z$-score $<-2$; and overweight/obesity: BMI-for-age $z$-score $>1$; these categories were not exclusive and overlapped in some cases (e.g., a child who was classified as underweight might also be classified as stunted). An aggregate indicator of malnutrition, the Composite Index of Anthropometric Failure (CIAF), was determined by identifying all children who were in anthropometric failure (i.e., all children who were (1) wasted only; (2) wasted and underweight; (3) wasted, stunted, and underweight; (4) stunted and underweight; (5) stunted only; or (6) underweight only; wasting, stunting, and underweight were defined according to the WHO classification as described above) while excluding those children who were not in anthropometric failure (i.e., children whose weight-for-age, height-for-age, and BMI-for-age $z$-scores were $\geq-2$ ) [22]. The CIAF identifies children who belong exclusively to one of these categories; therefore, it provides a single measure of the severity of undernutrition in the population [22]. A waist-to-height ratio (WHtR) was calculated as WC in $\mathrm{cm}$ divided by height in $\mathrm{cm}$. There are no specific WHtR cutoffs for the identification of overweight or obesity in Vietnamese children; therefore, we defined abdominal overweight/obesity as WHtR $\geq 0.46$ for boys and WHtR $\geq 0.45$ for girls according to the cutoffs developed by Nambiar et al. [23]. These cutoffs have been shown to correspond to the $\geq 85$ th percentile for percentage body fat (i.e., $\geq$ overweight) in a nationally representative sample of 2773 8-16-year-old Australian school children, which also included Asian children [23]. 


\subsection{Socioeconomic Data}

Mothers' current employment status, mothers' education level, and monthly household income data were collected from the mothers via a pretested self-administered questionnaire.

\subsection{Data Analysis}

Data were collected from 2334 children. Sex data were available for all children; weight data for 2333 children; age data for 2331 children; height, weight-for-age $z$-score, and CIAF data for 2330 children; WC data for 2329 children; height-for-age and BMI-for-age $z$-score data for 2327 children; and WHtR data for 2326 children. Seventy-one percent of mothers provided information on their current employment status $(n=1647)$, and their education level $(n=1652)$, and $68 \%$ of mothers $(n=1587)$ provided information on the monthly household income.

The prevalence of underweight, stunting, wasting, anthropometric failure, overweight/obesity, and abdominal overweight/obesity was estimated for the whole sample, and for the levels of the following factors: sex, age, mother's current employment status, mother's education level, and monthly household income.

Children's age was categorized into tertiles ( 69 to $<82$ months, 82 to $<93$ months, and 93 to $\leq 108$ months). Mothers' education level was classified into three categories: beyond high school, high school, and below high school. High school education comprises 3 years of formal schooling, from the 10th to 12th grade, and is normally the minimum qualification required to obtain employment in Vietnam. The average income in rural areas in Vietnam in 2014 was 4.1 million VND/two adults/month (i.e., 176 USD) [24]. This monthly household income was used as a cutoff to differentiate between below-average and above-average income. Monthly household income was classified into four categories: $<4.1$ million VND (i.e., $<176$ USD), 4.1 to $<8$ million VND (i.e., 176 to $<344$ USD), 8 to $<11$ million VND (i.e., 344 to $<472$ USD), and $\geq 11$ million VND (i.e., $\geq 472$ USD).

Generalised linear mixed models, with school as a random effect, were used to estimate the association between binary outcomes (underweight, stunting, wasting, anthropometric failure, overweight/obesity, and abdominal overweight/obesity) with demographic variables. We report univariate associations, i.e., only one demographic factor considered at a time, and adjusted associations estimated under models including all demographic factors (i.e., age, sex, mother's current employment status, mother's education level, and monthly household income) [25]. Continuous outcomes (BMI-for-age $z$-scores and WHtR) were analysed following the same approach but using linear mixed models. For each outcome, we also report a measure of similarity of outcomes among children attending the same school, the intracluster correlation coefficient, estimated under a model with school as a random effect and no covariates. All estimates are reported along with $95 \%$ confidence intervals (CI). Analyses were performed with Stata (version 14.0; StataCorp LP, TX, USA).

\section{Results}

\subsection{Participants}

A total of 3960 children were invited to take part in this study. Of these, 2334 children were eligible and agreed to participate. A similar proportion of girls and boys took part in this study (Table 1).

The majority of children's mothers currently engaged in farm work and nearly two thirds were educated to a high school level or beyond (Table 1). Among the mothers who were self-employed, $84.7 \%$ had a low level of education (i.e., below high school) (data not shown in tables). More than half of the participating children came from households with a mean monthly income of $\geq 8$ million VND, whereas nearly one in six children came from families with a mean monthly income below the average two-adult income in rural areas in Vietnam. Mothers from families with a below-average monthly income were more likely to have a low education level $(67.9 \%$ had an education level below high school) (data not shown in tables). 
Table 1. Characteristics of the study participants.

\begin{tabular}{|c|c|c|c|}
\hline \multicolumn{2}{|c|}{ All Children } & $N(2334)$ & $\%$ \\
\hline \multirow{2}{*}{ Sex } & Girls & 1179 & 50.5 \\
\hline & Boys & 1155 & 49.5 \\
\hline \multirow{3}{*}{ Age (months) } & 69 to $<82$ & 773 & 33.2 \\
\hline & 82 to $<93$ & 806 & 34.6 \\
\hline & 93 to $\leq 108$ & 752 & 32.3 \\
\hline \multirow{4}{*}{ Mother's current employment status } & Self-employed & 204 & 12.4 \\
\hline & Employed full time & 342 & 20.8 \\
\hline & Farmer & 970 & 58.9 \\
\hline & Unemployed & 131 & 8.0 \\
\hline \multirow{3}{*}{ Mother's education level } & Above high school & 465 & 28.1 \\
\hline & High school & 554 & 33.5 \\
\hline & Below high school & 633 & 38.3 \\
\hline \multirow{4}{*}{ Monthly household income (million VND) } & $<4.1$ & 255 & 16.1 \\
\hline & 4.1 to $<8$ & 501 & 31.6 \\
\hline & 8 to $<11$ & 624 & 39.3 \\
\hline & $\geq 11$ & 207 & 13.0 \\
\hline \multirow{8}{*}{ Subgroups of anthropometric failure ${ }^{b}$} & No anthropometric failure ${ }^{a}$ & 2053 & 88.1 \\
\hline & Composite Index of Anthropometric Failure & 277 & 11.9 \\
\hline & Wasting only & 60 & 2.6 \\
\hline & Wasting + underweight & 51 & 2.2 \\
\hline & Wasting + stunting + underweight & 13 & 0.6 \\
\hline & Stunting + underweight & 74 & 3.2 \\
\hline & Stunting only & 31 & 1.3 \\
\hline & Underweight only & 48 & 2.1 \\
\hline
\end{tabular}

a Weight-for-age, height-for-age, and BMI-for-age $z$-scores $\geq-2{ }^{\mathrm{b}}$ Subgroups were defined according to the WHO classification for wasting, underweight, and stunting [21].

\subsection{Prevalence of Undernutrition and Overnutrition, and Associations between Anthropometric Indicators and} Sociodemographic Factors

The prevalence of underweight was $8.0 \%$ (i.e., all these children were underweight, in addition, some children were also stunted, stunted and wasted, or wasted) with no significant difference between boys and girls, or across age or household income categories (Table 2). Mother's current employment status and education level were univariately associated with underweight. In the multivariate analysis including all sociodemographic factors, mother's education level was the only factor significantly associated with underweight. Children of mothers with low education (below high school) were more likely to be underweight compared with children whose mothers were educated beyond high school (Odds Ratio (OR) 3.60; 95\% CI: 1.95, 6.64).

Table 2. Prevalence of underweight (weight-for-age $z$-score $<-2$ ) and associated sociodemographic factors in 6-9-year-old children in rural areas of Vietnam.

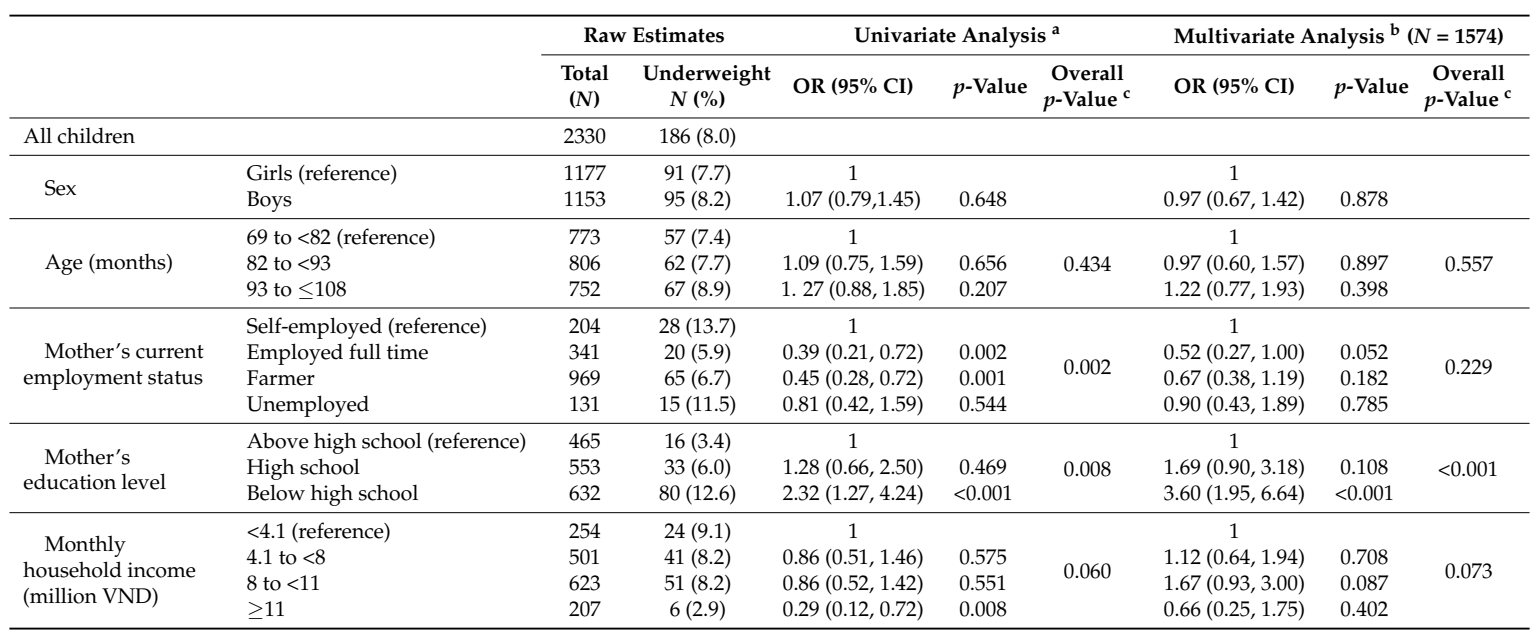

${ }^{a}$ Generalised linear mixed model including the covariate as a fixed effect and school as a random effect.

${ }^{b}$ Generalised linear mixed model including all five sociodemographic factors as fixed effects and school as a random effect. ${ }^{c}$ Overall $p$-value for the association between the outcome and the sociodemographic factor. 
The prevalence of stunting was 5.1\%. In univariate analyses, stunting was only associated with mother's education level (Table 3). After adjustment for all sociodemographic factors, there was no factor significantly associated with stunting.

Table 3. Prevalence of stunting (height-for-age $z$-score $<-2$ ) and associated sociodemographic factors in 6-9-year-old children in rural areas of Vietnam.

\begin{tabular}{|c|c|c|c|c|c|c|c|c|c|}
\hline & & \multicolumn{2}{|c|}{ Raw Estimates } & \multicolumn{3}{|c|}{ Univariate Analysis $^{\text {a }}$} & \multicolumn{3}{|c|}{ Multivariate Analysis ${ }^{b}(N=1570)$} \\
\hline & & $\begin{array}{l}\text { Total } \\
(N)\end{array}$ & $\begin{array}{l}\text { Stunting } \\
N(\%)\end{array}$ & OR $(95 \% \mathrm{CI})$ & $p$-Value & $\begin{array}{c}\text { Overall } \\
p \text {-Value } \\
{ }^{\mathrm{c}}\end{array}$ & OR $(95 \% \mathrm{CI})$ & $p$-Value & $\begin{array}{c}\text { Overall } \\
p \text {-Value }^{\mathrm{c}}\end{array}$ \\
\hline All children & & 2327 & $118(5.1)$ & & & & & & \\
\hline \multirow[b]{2}{*}{ Sex } & Girls (reference) & 1175 & $54(4.6)$ & 1 & & & 1 & & \\
\hline & Boys & 1152 & $64(5.6)$ & $1.22(0.84,1.77)$ & 0.295 & & $1.30(0.82,2.05)$ & 0.267 & \\
\hline \multirow{3}{*}{ Age (months) } & 69 to $<82$ (reference) & 773 & $34(4.4)$ & 1 & & \multirow{3}{*}{0.117} & 1 & & \multirow{3}{*}{0.100} \\
\hline & 82 to $<93$ & 806 & $36(4.5)$ & $1.03(0.64,1.68)$ & 0.893 & & $1.12(0.61,2.05)$ & 0.713 & \\
\hline & 93 to $\leq 108$ & 752 & $48(6.4)$ & $1.52(0.96,2.41)$ & 0.072 & & $1.77(1.00,3.13)$ & 0.052 & \\
\hline \multirow{4}{*}{$\begin{array}{r}\text { Mother's current } \\
\text { employment status }\end{array}$} & Self-employed (reference) & 204 & $15(7.4)$ & 1 & & \multirow{4}{*}{0.265} & 1 & & \multirow{4}{*}{0.594} \\
\hline & Employed full time & 340 & $14(4.1)$ & $0.53(0.25,1.13)$ & 0.103 & & $0.63(0.27,1.43)$ & 0.267 & \\
\hline & Farmer & 968 & $45(4.6)$ & $0.61(0.33,1.11)$ & 0.109 & & $0.77(0.37,1.59)$ & 0.477 & \\
\hline & Unemployed & 131 & $9(6.9)$ & $0.89(0.37,2.13)$ & 0.791 & & $1.07(0.44,2.66)$ & 0.882 & \\
\hline \multirow{3}{*}{$\begin{array}{l}\text { Mother's } \\
\text { education level }\end{array}$} & Above high school (reference) & 465 & $15(3.2)$ & 1 & & \multirow{3}{*}{0.008} & 1 & & \multirow{3}{*}{0.055} \\
\hline & High school & 553 & $23(4.2)$ & $1.28(0.66,2.49)$ & 0.471 & & $1.18(0.59,2.37)$ & 0.647 & \\
\hline & Below high school & 630 & $45(7.1)$ & $2.31(1.26,4.22)$ & 0.007 & & $2.05(1.03,4.04)$ & 0.040 & \\
\hline \multirow{4}{*}{$\begin{array}{l}\text { Monthly } \\
\text { household income } \\
\text { (million VND) }\end{array}$} & $<4.1$ (reference) & 254 & $13(5.1)$ & 1 & & \multirow{4}{*}{0.202} & 1 & & \multirow{4}{*}{0.143} \\
\hline & 4.1 to $<8$ & 500 & $27(5.4)$ & $1.03(0.52,2.05)$ & 0.930 & & $1.28(0.63,2.62)$ & 0.498 & \\
\hline & 8 to $<11$ & 622 & $36(5.8)$ & $1.10(0.57,2.13)$ & 0.778 & & $1.74(0.82,3.70)$ & 0.146 & \\
\hline & $\geq 11$ & 207 & $4(1.9)$ & $0.35(0.11,1.07)$ & 0.074 & & $0.60(0.18,2.04)$ & 0.417 & \\
\hline
\end{tabular}

${ }^{a}$ Generalised linear mixed model including the covariate as a fixed effect and school as a random effect.

b Generalised linear mixed model including all five sociodemographic factors as fixed effects and school as a

random effect. ${ }^{c}$ Overall $p$-value for the association between the outcome and the sociodemographic factor.

The prevalence of wasting was 5.3\% with no significant differences between sexes, or across the child age or mother's current employment status (Table 4). In univariate analyses, mother's education level and monthly household income were significantly associated with wasting; however, these associations were no longer significant after adjustment for other sociodemographic factors.

Table 4. Prevalence of wasting (BMI-for-age $z$-score $<-2$ ) and associated sociodemographic factors in 6-9-year-old children in rural areas of Vietnam.

\begin{tabular}{|c|c|c|c|c|c|c|c|c|c|}
\hline & & \multicolumn{2}{|c|}{ Raw Estimates } & \multicolumn{3}{|c|}{ Univariate Analysis $^{a}$} & \multicolumn{3}{|c|}{ Multivariate Analysis ${ }^{b}(N=1570)$} \\
\hline & & $\begin{array}{l}\text { Total } \\
(N)\end{array}$ & $\begin{array}{c}\text { Wasting } \\
N(\%)\end{array}$ & OR $(95 \% \mathrm{CI})$ & $p$-Value & $\begin{array}{c}\text { overall } \\
p \text {-Value }^{\mathrm{c}}\end{array}$ & OR $(95 \% \mathrm{CI})$ & $p$-Value & $\begin{array}{c}\text { Overall } \\
p \text {-Value }^{\mathrm{c}}\end{array}$ \\
\hline All children & & 2327 & $124(5.3)$ & & & & & & \\
\hline \multirow{2}{*}{ Sex } & Girls (reference) & 1175 & $65(5.5)$ & 1 & & & 1 & & \\
\hline & Boys & 1152 & $59(5.1)$ & $0.92(0.64,1.32)$ & 0.656 & & $1.30(0.82,2.05)$ & 0.267 & \\
\hline \multirow{3}{*}{ Age (months) } & 69 to $<82$ (reference) & 773 & $37(4.8)$ & 1 & & \multirow{3}{*}{0.209} & 1 & & \multirow{3}{*}{0.500} \\
\hline & 82 to $<93$ & 806 & $38(4.7)$ & $0.99(0.62,1.58)$ & 0.966 & & $0.76(0.43,1.35)$ & 0.351 & \\
\hline & 93 to $\leq 108$ & 752 & $49(6.5)$ & $1.39(0.89,2.17)$ & 0.143 & & $1.05(0.61,1.81)$ & 0.851 & \\
\hline \multirow{4}{*}{$\begin{array}{l}\text { Mother's current } \\
\text { employment status }\end{array}$} & Self-employed (reference) & 204 & $17(8.3)$ & 1 & & \multirow{4}{*}{0.234} & 1 & & \multirow{4}{*}{0.592} \\
\hline & Employed full time & 340 & $17(5.0)$ & $0.58(0.29,1.16)$ & 0.126 & & $0.94(0.44,2.04)$ & 0.885 & \\
\hline & Farmer & 968 & $46(4.7)$ & $0.55(0.31,0.98)$ & 0.042 & & $0.99(0.50,2.00)$ & 0.998 & \\
\hline & Unemployed & 131 & $7(5.3)$ & $0.62(0.25,1.54)$ & 0.304 & & $0.49(0.15,1.54)$ & 0.222 & \\
\hline \multirow{3}{*}{$\begin{array}{l}\text { Mother's } \\
\text { education level }\end{array}$} & Above high school (reference) & 465 & $14(3.0)$ & 1 & & \multirow{3}{*}{0.008} & 1 & & \multirow{3}{*}{0.250} \\
\hline & High school & 553 & $27(4.9)$ & $1.65(0.86,3.19)$ & 0.134 & & $1.42(0.71,2.83)$ & 0.317 & \\
\hline & Below high school & 630 & $46(7.3)$ & $2.54(1.38,4.68)$ & 0.003 & & $1.80(0.90,3.63)$ & 0.098 & \\
\hline \multirow{4}{*}{$\begin{array}{l}\text { Monthly } \\
\text { household income } \\
\text { (million VND) }\end{array}$} & $<4.1$ (reference) & 254 & $19(7.5)$ & 1 & & \multirow{4}{*}{0.031} & 1 & & \multirow{4}{*}{0.227} \\
\hline & 4.1 to $<8$ & 500 & $32(6.4)$ & $0.85(0.47,1.53)$ & 0.582 & & $0.94(0.51,1.75)$ & 0.853 & \\
\hline & 8 to $<11$ & 622 & $22(3.5)$ & $0.45(0.24,0.85)$ & 0.014 & & $0.54(0.27,1.12)$ & 0.098 & \\
\hline & $\geq 11$ & 207 & $7(3.3)$ & $0.43(0.18,1.05)$ & 0.064 & & $0.56(0.22,1.48)$ & 0.243 & \\
\hline
\end{tabular}

${ }^{a}$ Generalised linear mixed model including the covariate as a fixed effect and school as a random effect.

b Generalised linear mixed model including all five sociodemographic factors as fixed effects and school as a random effect. ${ }^{c}$ Overall $p$-value for the association between the outcome and the sociodemographic factor.

The aggregate prevalence of undernutrition as measured by the CIAF indicated that $11.9 \%$ of all children were in anthropometric failure (Table 5). Anthropometric failure was significantly associated in univariate analyses with child age, mother's current employment status, mother's education level, and monthly household income. However, in the multivariate analysis, only mother's education level was associated with anthropometric failure. Children whose mothers' education level was below high 
school were more likely to be in anthropometric failure compared with children whose mothers were educated beyond high school (OR 2.00, 95\% CI: 1.26, 3.19).

Table 5. Prevalence of anthropometric failure and associated sociodemographic factors in 6-9-year-old children in rural areas of Vietnam.

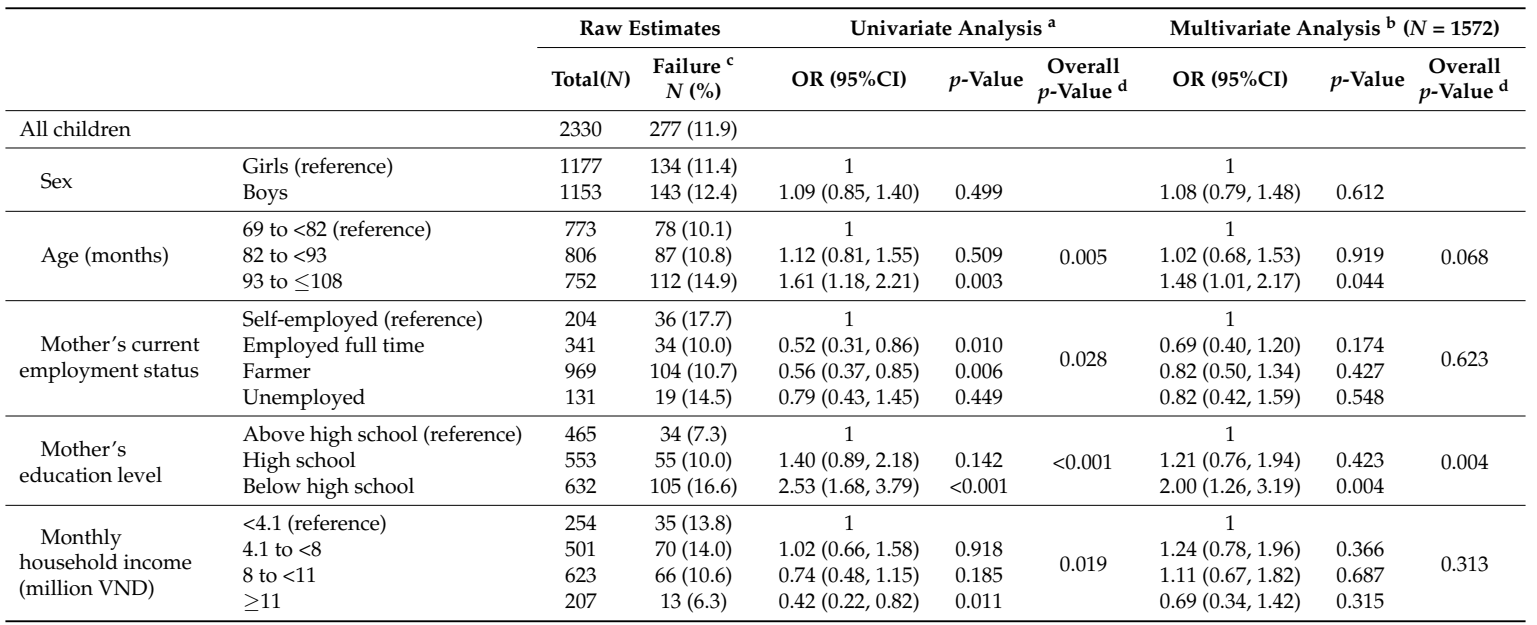

${ }^{a}$ Generalised linear mixed model including the covariate as a fixed effect and school as a random effect.

$\mathrm{b}$ Generalised linear mixed model including all five sociodemographic factors as fixed effects and school as a random effect. ' Composite Index of Anthropometric Failure; weight-for-age, height-for-age, and/or BMI-for-age $z$-scores $<-2 .{ }^{\mathrm{d}}$ Overall $\mathrm{p}$-value for the association between the outcome and the sociodemographic factor.

The prevalence of overweight/obesity was $22.1 \%$, and it was higher in boys than girls $(27.2 \%$ vs. $17.1 \% ; p<0.001$; Table 6). Overweight/obesity was also significantly associated in the univariate analyses with mother's current employment status, mother's education level, and monthly household income. However, in the multivariate analysis only child sex and mother's education level remained significantly associated with overweight/obesity. In comparison with children whose mothers had an education level above high school, children whose mothers had high school or below high school education were significantly less likely to be overweight/obese (OR 0.59, 95\% CI: $0.44,0.81$, and OR 0.37, 95\% CI: 0.26, 0.53, respectively; Table 6). Similar results were obtained when BMI-for-age $z$-scores were analysed (Table 7).

Table 6. Prevalence of overweight/obesity (BMI-for-age $z$-score $>1$ ) and associated sociodemographic factors in 6-9-year-old children in rural areas of Vietnam.

\begin{tabular}{|c|c|c|c|c|c|c|c|c|c|}
\hline & & \multicolumn{2}{|c|}{ Raw Estimates } & \multicolumn{3}{|c|}{ Univariate Analysis $^{\text {a }}$} & \multicolumn{3}{|c|}{ Multivariate Analysis $^{\mathrm{b}}(N=1570)$} \\
\hline & & $\operatorname{Total}(N)$ & $\begin{array}{l}\text { Overweight/ } \\
\text { Obesity } N(\%)\end{array}$ & OR $(95 \% \mathrm{CI})$ & $p$-Value & $\begin{array}{l}\text { Overall } \\
p \text {-Value }\end{array}$ & OR $(95 \% \mathrm{CI})$ & $p$-Value & $\begin{array}{l}\text { Overall } \\
p \text {-Value } \\
\end{array}$ \\
\hline All children & & 2327 & $514(22.1)$ & & & & & & \\
\hline \multirow[b]{2}{*}{ Sex } & Girls (reference) & 1175 & $201(17.1)$ & 1 & & & 1 & & \\
\hline & Boys & 1152 & $313(27.2)$ & $1.83(1.50,2.25)$ & $<0.001$ & & $2.04(1.58,2.61)$ & $<0.001$ & \\
\hline \multirow{3}{*}{ Age (months) } & 69 to $<82$ (reference) & 773 & $150(19.5)$ & 1 & & \multirow{3}{*}{0.146} & 1 & & \multirow{3}{*}{0.071} \\
\hline & 82 to $<93$ & 806 & $197(24.5)$ & $1.28(1.00,1.63)$ & 0.050 & & $1.41(1.05,1.90)$ & 0.023 & \\
\hline & 93 to $\leq 108$ & 752 & $167(22.2)$ & $1.16(0.90,1.49)$ & 0.253 & & $1.16(0.84,1.60)$ & 0.368 & \\
\hline \multirow{4}{*}{$\begin{array}{r}\text { Mother's current } \\
\text { employment status }\end{array}$} & Self-employed (reference) & 204 & $29(14.2)$ & 1 & & \multirow{4}{*}{0.022} & 1 & & \multirow{4}{*}{0.647} \\
\hline & Employed full time & 340 & $82(24.1)$ & $1.80(1.12,2.88)$ & 0.015 & & $1.19(0.70,2.03)$ & 0.528 & \\
\hline & Farmer & 968 & $221(22.8)$ & $1.66(1.09,2.55)$ & 0.020 & & $0.97(0.59,1.62)$ & 0.920 & \\
\hline & Unemployed & 131 & $20(15.3)$ & $1.02(0.54,1.88)$ & 0.959 & & $0.96(0.49,1.87)$ & 0.894 & \\
\hline \multirow{3}{*}{$\begin{array}{l}\text { Mother's } \\
\text { education level }\end{array}$} & Above high school (reference) & 465 & 147 (31.6) & 1 & & \multirow{3}{*}{$<0.001$} & 1 & & \multirow{3}{*}{$<0.001$} \\
\hline & High school & 553 & $117(21.2)$ & $0.58(0.44,0.77)$ & $<0.001$ & & $0.59(0.44,0.81)$ & 0.001 & \\
\hline & Below high school & 630 & $89(14.1)$ & $0.36(0.26,0.48)$ & $<0.001$ & & $0.37(0.26,0.53)$ & $<0.001$ & \\
\hline \multirow{4}{*}{$\begin{array}{l}\text { Monthly } \\
\text { household income } \\
\text { (million VND) }\end{array}$} & $<4.1$ (reference) & 254 & 37 (14.6) & 1 & & \multirow{4}{*}{0.003} & 1 & & \multirow{4}{*}{0.561} \\
\hline & 4.1 to $<8$ & 500 & $92(18.4)$ & $1.31(0.86,1.99)$ & 0.208 & & $1.12(0.72,1.73)$ & 0.620 & \\
\hline & 8 to $<11$ & 622 & $154(24.8)$ & $1.86(1.25,2.78)$ & 0.002 & & $1.30(0.84,2.02)$ & 0.239 & \\
\hline & $\geq 11$ & 207 & $56(27.1)$ & $2.02(1.25,3.26)$ & 0.004 & & $1.35(0.80,2.27)$ & 0.256 & \\
\hline
\end{tabular}

a Generalised linear mixed model including the covariate as a fixed effect and school as a random effect.

b Generalised linear mixed model including all five sociodemographic factors as fixed effects and school as a random effect. ${ }^{c}$ Overall $p$-value for the association between the outcome and the sociodemographic factor. 
The prevalence of abdominal overweight/obesity was 31.0\% (Table 8). In univariate analyses, abdominal overweight/obesity was significantly associated with sex, mother's current employment status, mother's education level, and monthly household income. In the multivariate model, only sex and mother's education level were significantly associated with abdominal overweight/obesity, with a higher risk of abdominal overweight/obesity in boys compared with girls (OR 1.55, 95\% CI: $1.24,1.93)$, and a lower risk in children whose mothers' education level was at or below high school compared with children whose mothers were educated beyond high school (OR 0.65, 95\% CI: 0.49, 0.86 and OR $0.47,95 \%$ CI: $0.35,0.65$, respectively). Additionally, WHtR was higher in boys than girls, lower in the oldest children compared with the youngest children, and lower in children of mothers with education at or below high school compared with children of mothers with education above high school (Table 9).

Table 7. BMI-for-age z-scores and associated sociodemographic factors in 6-9-year-old children in rural areas of Vietnam.

\begin{tabular}{|c|c|c|c|c|c|c|c|c|c|c|}
\hline & & \multicolumn{3}{|c|}{ Raw Estimates } & \multicolumn{3}{|c|}{ Univariate Analysis $^{\text {a }}$} & \multicolumn{3}{|c|}{ Multivariate Analysis ${ }^{\mathrm{b}}(N=1570)$} \\
\hline & & $\begin{array}{c}\text { Total } \\
(N)\end{array}$ & Mean & SE & $\beta(95 \% \mathrm{CI})$ & $p$-Value & $\begin{array}{l}\text { Overall } \\
p \text {-Value }^{\mathrm{c}}\end{array}$ & $\beta(95 \% \mathrm{CI})$ & $p$-Value & $\begin{array}{c}\text { Overall } \\
p \text {-Value }\end{array}$ \\
\hline All children & & 2327 & -0.103 & 0.029 & & & & & & \\
\hline Age (months) & $\begin{array}{l}69 \text { to }<82 \text { (reference) } \\
82 \text { to }<93 \\
93 \text { to } \leq 108\end{array}$ & $\begin{array}{l}773 \\
806 \\
752\end{array}$ & $\begin{array}{l}-0.116 \\
-0.035 \\
-0.157\end{array}$ & $\begin{array}{l}0.105 \\
0.104 \\
0.104\end{array}$ & $\begin{array}{c}0.080(-0.057,0.218) \\
-0.042(-0.182,0.099)\end{array}$ & $\begin{array}{l}0.251 \\
0.560\end{array}$ & 0.206 & $\begin{array}{l}0.153(-0.009,0.316) \\
0.001(-0.167,0.170)\end{array}$ & $\begin{array}{l}0.065 \\
0.991\end{array}$ & 0.106 \\
\hline $\begin{array}{l}\text { Mother's } \\
\text { education level }\end{array}$ & $\begin{array}{l}\text { Above high school (reference) } \\
\text { High school } \\
\text { Below high school }\end{array}$ & $\begin{array}{l}465 \\
553 \\
630 \\
\end{array}$ & $\begin{array}{l}0.251 \\
-0.072 \\
-0.405 \\
\end{array}$ & $\begin{array}{l}0.081 \\
0.077 \\
0.074 \\
\end{array}$ & $\begin{array}{l}-0.322(-0.494,-0.150) \\
-0.656(-0.824,-0.487)\end{array}$ & $\begin{array}{l}<0.001 \\
<0.001\end{array}$ & $<0.001$ & $\begin{array}{l}-0.281(-0.460,-0.101) \\
-0.577(-0.770,-0.383)\end{array}$ & $\begin{array}{l}0.002 \\
<0.001 \\
\end{array}$ & $<0.001$ \\
\hline $\begin{array}{l}\text { Monthly } \\
\text { household income } \\
\text { (million VND) }\end{array}$ & $\begin{array}{l}<4.1 \text { (reference) } \\
4.1 \text { to }<8 \\
8 \text { to }<11 \\
\geq 11\end{array}$ & $\begin{array}{l}254 \\
500 \\
622 \\
207\end{array}$ & $\begin{array}{l}-0.329 \\
-0.224 \\
0.016 \\
0.131\end{array}$ & $\begin{array}{l}0.114 \\
0.096 \\
0.092 \\
0.121\end{array}$ & $\begin{array}{c}0.105(-0.105,0.316) \\
0.345(0.140,0.550) \\
0.460(0.202,0.719)\end{array}$ & $\begin{array}{l}0.327 \\
0.001 \\
<0.001\end{array}$ & $<0.001$ & $\begin{array}{c}-0.036(-0.249,0.178) \\
0.070(-0.152,0.292) \\
0.147(-0.127,0.422\end{array}$ & $\begin{array}{l}0.744 \\
0.537 \\
0.293\end{array}$ & 0.422 \\
\hline
\end{tabular}

${ }^{a}$ Linear mixed model including the covariate as a fixed effect and school as a random effect. ${ }^{\mathrm{b}}$ Linear mixed model including all five sociodemographic factors as fixed effects and school as a random effect. ${ }^{c}$ Overall $p$-value for the association between the outcome and the sociodemographic factor.

Table 8. Prevalence of abdominal overweight/obesity (WHtR $\geq 0.46$ for boys and $\geq 0.45$ for girls) and associated sociodemographic factors in 6-9-year-old children in rural areas of Vietnam.

\begin{tabular}{|c|c|c|c|c|c|c|c|c|c|}
\hline & & \multicolumn{2}{|c|}{ Raw Estimates } & \multicolumn{3}{|c|}{ Univariate Analysis a } & \multicolumn{3}{|c|}{ Multivariate Analysis $^{b}(N=1567)$} \\
\hline & & $\operatorname{Total}(N)$ & $\begin{array}{c}\text { Abdominal } \\
\text { Overweight/ } \\
\text { Obesity } N(\%)\end{array}$ & OR $(95 \% \mathrm{CI})$ & $p$-Value & $\begin{array}{l}\text { Overall } \\
p \text {-Value }\end{array}$ & OR $(95 \% \mathrm{CI})$ & $p$-Value & $\begin{array}{l}\text { Overall } \\
p \text {-Value } \\
{ }^{\mathrm{c}}\end{array}$ \\
\hline All children & & 2326 & $721(31.0)$ & & & & & & \\
\hline \multirow{2}{*}{ Sex } & Girls (reference) & 1174 & $325(27.7)$ & 1 & & & 1 & & \\
\hline & Boys & 1152 & $396(34.4)$ & $1.37(1.15,1.64)$ & $<0.001$ & & $1.55(1.24,1.93)$ & $<0.001$ & \\
\hline \multirow{3}{*}{ Age (months) } & 69 to $<82$ (reference) & 773 & $260(33.9)$ & 1 & & \multirow{3}{*}{0.070} & 1 & & \multirow{3}{*}{0.180} \\
\hline & 82 to $<93$ & 806 & $246(30.6)$ & $0.83(0.67,1.03)$ & 0.092 & & $0.94(0.73,1.22)$ & 0.646 & \\
\hline & 93 to $\leq 108$ & 752 & $215(28.6)$ & $0.78(0.62,0.97)$ & 0.028 & & $0.78(0.59,1.02)$ & 0.072 & \\
\hline \multirow{4}{*}{$\begin{array}{l}\text { Mother's current } \\
\text { employment status }\end{array}$} & Self-employed (reference) & 204 & $44(21.6)$ & 1 & & \multirow{4}{*}{0.021} & 1 & & \multirow{4}{*}{0.876} \\
\hline & Employed full time & 341 & $110(32.3)$ & $1.67(1.11,2.51)$ & 0.014 & & $1.19(0.75,1.87)$ & 0.459 & \\
\hline & Farmer & 966 & $316(32.7)$ & $1.70(1.18,2.45)$ & 0.004 & & $1.09(0.71,1.67)$ & 0.700 & \\
\hline & Unemployed & 131 & $34(26.0)$ & $1.25(0.75,2.11)$ & 0.389 & & $1.06(0.61,1.85)$ & 0.829 & \\
\hline \multirow{3}{*}{$\begin{array}{l}\text { Mother's } \\
\text { education level }\end{array}$} & Above high school (reference) & 463 & $193(41.7)$ & 1 & & \multirow{3}{*}{$<0.001$} & 1 & & \multirow{3}{*}{$<0.001$} \\
\hline & High school & 553 & $167(30.2)$ & $0.61(0.47,0.78)$ & $<0.001$ & & $0.65(0.49,0.86)$ & 0.003 & \\
\hline & Below high school & 631 & $146(23.1)$ & $0.42(0.32,0.55)$ & $<0.001$ & & $0.47(0.35,0.65)$ & $<0.001$ & \\
\hline \multirow{4}{*}{$\begin{array}{l}\text { Monthly } \\
\text { household income } \\
\text { (million VND) }\end{array}$} & $<4.1$ (reference) & 255 & $59(23.1)$ & 1 & & \multirow{4}{*}{0.009} & 1 & & \multirow{4}{*}{0.772} \\
\hline & 4.1 to $<8$ & 499 & $144(28.9)$ & $1.34(0.94,1.90)$ & 0.102 & & $1.16(0.80,1.68)$ & 0.426 & \\
\hline & 8 to $<11$ & 621 & $211(34.0)$ & $1.68(1.19,2.36)$ & 0.003 & & $1.22(0.84,1.78)$ & 0.293 & \\
\hline & $\geq 11$ & 207 & $74(35.8)$ & $1.80(1.19,2.72)$ & 0.005 & & $1.20(0.76,1.88)$ & 0.420 & \\
\hline
\end{tabular}

a Generalised linear mixed model including the covariate as a fixed effect and school as a random effect.

${ }^{\mathrm{b}}$ Generalised linear mixed model including all five sociodemographic factors as fixed effects and school as a random effect. ${ }^{\mathrm{c}}$ Overall $p$-value for the association between the outcome and the sociodemographic factor. 
Table 9. Waist-to-Height Ratio and associated socio-demographic factors in 6-9-year-old children in rural areas of Vietnam.

\begin{tabular}{|c|c|c|c|c|c|c|c|c|c|c|}
\hline & & \multicolumn{3}{|c|}{ Raw Estimates } & \multicolumn{3}{|c|}{ Univariate Analysis a $^{\text {a }}$} & \multicolumn{3}{|c|}{ Multivariate Analysis $^{\mathrm{b}}(N=1567)$} \\
\hline & & $\begin{array}{c}\text { Total } \\
(N)\end{array}$ & Mean & SE & $\beta(95 \% \mathrm{CI})$ & $p$-Value & $\begin{array}{l}\text { Overall } \\
p \text {-Value }\end{array}$ & $\beta(95 \% \mathrm{CI})$ & $p$-Value & $\begin{array}{c}\text { Overall } \\
p \text {-Value }\end{array}$ \\
\hline All children & & 2326 & 0.444 & 0.001 & & & & & & \\
\hline \multirow[b]{2}{*}{ Sex } & Girls (reference level) & 1174 & 0.434 & 0.003 & & & & & & \\
\hline & Boys & 1152 & 0.452 & 0.003 & $0.018(0.015,0.022)$ & $<0.001$ & & $0.020(0.016,0.025)$ & $<0.001$ & \\
\hline \multirow{3}{*}{ Age (months) } & 69 to $<82$ (reference) & 773 & 0.448 & 0.003 & & & \multirow{3}{*}{0.002} & & & \multirow{3}{*}{0.006} \\
\hline & 82 to $<93$ & 806 & 0.443 & 0.003 & $-0.004(-0.009,-0.000)$ & 0.048 & & $-0.001(-0.008,0.002)$ & 0.236 & \\
\hline & 93 to $\leq 108$ & 752 & 0.439 & 0.003 & $-0.008(-0.013,-0.004)$ & $<0.001$ & & $-0.009(-0.014,-0.003)$ & 0.001 & \\
\hline \multirow{4}{*}{$\begin{array}{r}\text { Mother's current } \\
\text { employment status }\end{array}$} & Self-employed (reference level) & 204 & 0.436 & 0.004 & & & \multirow{4}{*}{0.037} & & & \multirow{4}{*}{0.876} \\
\hline & Employed full time & 341 & 0.445 & 0.003 & $0.009(0.001,0.017)$ & 0.020 & & $0.003(-0.006,0.011)$ & 0.545 & \\
\hline & Farmer & 966 & 0.444 & 0.002 & $0.009(0.002,0.015)$ & 0.012 & & $0.000(-0.007,0.008)$ & 0.910 & \\
\hline & Unemployed & 131 & 0.438 & 0.004 & $0.003(-0.007,0.012)$ & 0.607 & & $0.002(-0.009,0.012)$ & 0.764 & \\
\hline \multirow{3}{*}{$\begin{array}{l}\text { Mother's } \\
\text { education level }\end{array}$} & Above high school (reference level) & 463 & 0.453 & 0.002 & & & \multirow{3}{*}{$<0.001$} & & & \multirow{3}{*}{$<0.001$} \\
\hline & High school & 553 & 0.442 & 0.002 & $-0.010(-0.016,-0.005)$ & $<0.001$ & & $-0.009(-0.015,-0.003)$ & 0.002 & \\
\hline & Below high school & 631 & 0.436 & 0.002 & $-0.017(-0.022,-0.011)$ & $<0.001$ & & $-0.014(-0.021,-0.008)$ & $<0.001$ & \\
\hline \multirow{4}{*}{$\begin{array}{l}\text { Monthly } \\
\text { household income } \\
\text { (million VND) }\end{array}$} & $<4.1$ (reference level) & 255 & 0.436 & 0.003 & & & \multirow{4}{*}{0.006} & & & \multirow{4}{*}{0.588} \\
\hline & 4.1 to $<8$ & 499 & 0.440 & 0.003 & $0.004(-0.003,0.011)$ & 0.283 & & $0.000(-0.007,0.007)$ & 0.981 & \\
\hline & 8 to $<11$ & 621 & 0.447 & 0.002 & $0.010(0.004,0.017)$ & 0.002 & & $0.004(-0.003,0.011)$ & 0.315 & \\
\hline & $\geq 11$ & 207 & 0.446 & 0.003 & $0.010(0.001,0.018)$ & 0.021 & & $0.002(-0.007,0.011)$ & 0.693 & \\
\hline
\end{tabular}

Finally, we present in Table 10 the estimated intracluster correlation coefficient for each of the outcomes assessed in this study. These estimates may inform the design and sample size calculations in future studies targeting the same outcomes in school children from rural areas in Vietnam.

Table 10. Estimates of the intracluster correlation coefficient (ICC) for each outcome measure ${ }^{\text {a }}$

\begin{tabular}{|c|c|}
\hline & ICC $(95 \% \mathrm{CI})$ \\
\hline Underweight $^{b}$ & $0.013(0.001,0.116)$ \\
\hline Stunting ${ }^{c}$ & $0.008(0.000,0.349)$ \\
\hline Wasting $\mathrm{d}$ & $0.001(0.000,0.999)$ \\
\hline Overweight/obesity e & $0.038(0.012,0.113)$ \\
\hline Abdominal overweight/obesity $\mathrm{f}$ & $0.020(0.006,0.068)$ \\
\hline Composite Index of Anthropometry Failure $\mathrm{g}$ & $0.009(0.001,0.091)$ \\
\hline BMI-for-age $z$-score & $0.034(0.012,0.096)$ \\
\hline Waist-to-Height Ratio & $0.019(0.006,0.061)$ \\
\hline
\end{tabular}

${ }^{a}$ ICC estimated under a mixed model with school as a random effect and no covariates. ${ }^{\mathrm{b}}$ Weight-for-age $z$-score $<-2$.

${ }^{\mathrm{c}}$ Height-for-age $z$-score $<-2 .{ }^{\mathrm{d}}$ BMI-for-age $z$-score $<-2$. ${ }^{\mathrm{e}}$ BMI-for-age $z$-score $>1$. ${ }^{\mathrm{f}}$ Waist-to-Height Ratio $\geq 0.46$

for boys and $\geq 0.45$ for girls. ${ }^{\mathrm{g}}$ Weight-for-age, height-for-age, and/or BMI-for-age $z$-scores $<-2$.

\section{Discussion}

\subsection{Prevalence of Undernutrition and Overnutrition}

This study provides evidence for the double burden of disease in primary school children in rural areas of Hai Phong City, Vietnam with nearly 12\% of children affected by anthropometric failure, $22 \%$ by overweight/obesity, and $31 \%$ by abdominal overweight/obesity.

We found that $8 \%$ of primary school children in rural areas in Hai Phong City in Vietnam were underweight. This confirms the results of our earlier cross-sectional study in 1004 primary school children in rural and urban areas of Hai Phong City in Vietnam which found $8.2 \%$ of children to be underweight [26].

Vietnam has been identified as one of 36 nations with the highest prevalence of stunting in the world [27]. In the present study, we found that $5.1 \%$ of children were at risk of stunting, which is similar to the prevalence of $7.6 \%$ found in our earlier cross-sectional study [26], or $2.4 \%$ reported recently in 3,000 primary school children in Hanoi City [28]. Compared with previous studies in other cities in Vietnam, wasting appeared less prevalent among primary school children in rural areas of Hai Phong City. Approximately 14\% of primary school children in 6 provinces in Vietnam were affected by wasting in 2011 [14] compared with 5.3\% children in our study. However, this proportion of children 
at risk of wasting has apparently not changed in the last four years as we also found that 5.3\% of school children were wasted in our earlier cross-sectional study conducted in the same city in 2012 [26]. Hai Phong City is a mega city in Vietnam (population of approximately 2 million people in 2016) with better socioeconomic conditions compared to other regions of Vietnam [29], which could be at least partially responsible for the lower risk of wasting.

A study conducted in 2011 in 2,872 children in 6 provinces in Vietnam found that $6.5 \%$ of school children in rural areas were affected by overweight and/or obesity [14]. In 2012, we found that $12 \%$ of school children in rural areas of Hai Phong City were affected by overweight or obesity [26]. In the current study, however, this prevalence nearly doubled as $22 \%$ of children were affected. This sharp increase in overweight and obesity in Hai Phong City over the last four years indicates that children in rural areas of Vietnam are at similar risk of excessive body weight as those residing in urban areas where a third of children are affected $[13,14,30]$. Improved socioeconomic status, poor diet, and inadequate physical inactivity may be partially responsible for this high increase in the prevalence of overweight and obesity in the rural areas of Hai Phong City [30,31]. Additionally, Vietnamese households have been shown to be susceptible to the marketing of energy-dense nutrient-poor foods [32]. Advertising of food products has been identified to affect food preferences, purchases, consumption [33], and food choices of children [34]. Marketing of energy-dense nutrient-poor foods, therefore, may contribute to childhood obesity [35].

\subsection{Associations between Sociodemographic Factors and Anthropometric Indicators}

This study revealed a significant and consistent relationship between maternal education and both undernutrition and overnutrition, and an association between sex and overnutrition.

Indeed, we found that low maternal education was a risk factor for underweight and anthropometric failure. This is consistent with findings from studies in Vietnam [14,36] and other lowand middle-income countries $[37,38]$. However, no significant association between undernutrition and maternal employment status or monthly household income was detected after adjusting for other sociodemographic variables including education. This is in contrast with other studies, which reported a significant negative correlation between income and malnutrition [37-39]. It is possible that we did not detect an association between household income and undernutrition in our study because the majority of families (i.e., 83.9\%) reported monthly income at or above the average two-adult income in rural areas of Vietnam, and only four families reported income at or below the poverty line of 0.4 million VND per month as defined by the Vietnamese Ministry of Labour, Invalids, and Social Affairs.

While undernutrition has been shown to be positively associated in rural areas with male sex [14] and age [39], we did not detect any association. Only in our univariate model age was associated with anthropometric failure, with older children at greater risk. Suboptimal habitual food consumption patterns and practices over the long term may be responsible for this trend [40]; however, our study did not explore this relationship. Similar to our research, a large study in Vietnam conducted in 2872 children found no relationship between undernutrition and child sex or age except for stunting, which was significantly higher in rural boys compared with girls (20.6\% vs. 14.7\%) [14].

Similar to undernutrition, we also found an association between overnutrition (i.e., overweight/ obesity and abdominal overweight/obesity) and maternal education; however, this association was positive. Children of mothers with an education level above high school were at highest risk of overweight/obesity. This is consistent with findings from our earlier cross-sectional study in 276 primary school children in rural and urban areas of Hai Phong City [25] but in contrast with research conducted in other provinces/cities in Vietnam in which the education level of parents was protective against childhood overweight and obesity [41,42]. We have speculated that in Hai Phong City, women who achieved a higher education level could find better jobs resulting in less time spent preparing and providing healthier meals for children (for example, greater reliance on fast foods or processed foods) leading to a higher risk of childhood overweight/obesity [25]. Our current study also supports the 
suggestion of Lindsay et al. [43] that childhood obesity prevention programmes should focus on parental roles.

In addition, our research showed that the prevalence of overnutrition was significantly different between sexes with boys at a higher risk of overweight/obesity and abdominal overweight/obesity compared with girls. This is consistent with previous findings in Vietnam [14,25]. In Vietnam, communities typically prioritise the needs of boys compared with girls [44], which could result in decreased expectations of boys to contribute to household chores, and instead increasing their time spent in physical inactivity, and hence risk of overweight/obesity. Furthermore, although we did not collect dietary data in the current study, our earlier research showed that boys in Hai Phong City consumed a greater variety of foods than girls [25], which has been previously shown to be a risk factor for increased energy intake and adiposity [45]. Although this study focused only on anthropometric data in association with selected socioeconomic factors, it is clear that programmes aiming to reduce overweight/obesity among school children in Vietnam should take into account the higher risk of overweight/obesity in boys.

The study's strengths include a random selection of the study districts and a large sample size. Additionally, all measurements were performed by well-trained staff from the National Institute of Nutrition in Vietnam to ensure high quality of data. However, as this was a cross-sectional study, it is difficult to detect temporal associations between undernutrition or overnutrition and the selected sociodemographic factors. Although more than 2000 children participated in this survey and the study sample was likely to be representative of all children attending primary schools in rural areas of Hai Phong City [26], it is unlikely that these children were representative of all primary school children in Vietnam [36,46]. In addition, as there are no specific WHtR cutoffs for the identification of abdominal overweight or obesity in Vietnamese children, we used the cutoffs by Nambiar et al. [23], which were developed for Australian children. However, there is still limited evidence on the most appropriate WHtR cutoffs for identifying abdominal overweight/obesity in Asian children, and those currently proposed are based on BMI rather than body fat [47,48].

\section{Conclusions}

In summary, undernutrition continues to be a public health problem in primary school children in rural areas in Hai Phong City affecting more than one in ten children. Overweight/obesity appears to have increased in the last four years with up to a third of children currently at risk. Low maternal education level was found to be a risk factor for underweight or anthropometric failure, whereas being male and high maternal education level was found to be a risk factor for overweight/obesity and abdominal overweight/obesity. Future interventions for the prevention and control of childhood obesity should take into consideration the difference in adiposity between boys and girls, and the need to target children of well-educated mothers. Programmes aimed at preventing child undernutrition need to emphasize the needs of children of mothers with low education.

Author Contributions: N.T.D.H., E.A.S.-G., T.D.L., R.S.G., A.F.W., and A.J.S., conceived the study; N.T.D.H., E.A.S.-G., L.O., R.S.G., A.F.W., and A.J.S. designed the study; T.D.L. obtained funding for the study; N.T.D.H., T.D.L., and E.A.S.-G. were responsible for data collection; N.T.D.H., E.A.S.-G., L.O., and T.D.L. developed the data analysis plan with contributions from R.S.G.; N.T.D.H. analysed the data with contributions from L.O. and E.A.S.-G.; N.T.D.H., E.A.S.-G., and L.O. interpreted data analysis; N.T.D.H. wrote the first draft of the manuscript with contributions from E.A.S.-G., L.O., and R.S.G.; N.T.D.H. and E.A.S.-G. took primary responsibility for the final content. All authors provided a critical review of the manuscript, and read and approved the final manuscript.

Funding: This research was funded by the National Institute of Nutrition, Vietnam. N.T.D.H. was supported by a Deakin University Postgraduate Research Scholarship.

Acknowledgments: The authors would like to acknowledge the National Institute of Nutrition in Vietnam, Hai Phong Preventive Medicine Centre, and the school teachers for their assistance with data collection; and thank participating children and parents.

Conflicts of Interest: The authors declared no potential conflict of interest. 


\section{References}

1. United Nations Development Programme. Discussion Paper: Addressing the Social Determinants of Noncommunicable Diseases. 2013. Available online: http://www.undp.org/content/dam/undp/library/ hivaids/English/Discussion_Paper_Addressing_the_Social_Determinants_of_NCDs_UNDP_2013.pdf (accessed on 3 August 2018).

2. United Nations Children's Fund. The Faces of Malnutrition. Available online: https://www.unicef.org/ nutrition/index_faces-of-malnutrition.html (accessed on 28 April 2015).

3. World Health Organization. Global and Regional Trends by UN Regions, 1990-2025, in Global Health Observatory Data Repository. Available online: http://apps.who.int/gho/data/view.main.NUTUNOVERWEIGHTv?lang= en (accessed on 28 March 2018).

4. Amare, B.; Moges, B.; Fantahun, B.; Tafess, K.; Woldeyohannes, D.; Yismaw, G.; Ayane, T.; Yabutani, T.; Mulu, A.; Ota, F.; et al. Micronutrient levels and nutritional status of school children living in Northwest Ethiopia. Nutr. J. 2012, 11. [CrossRef] [PubMed]

5. Das, P.; Basu, M.; Dhar, G.; Mallik, S.; Pal, R. Nutritional status and morbidity pattern of government primary school children in north Kolkata of West Bengal, India. South East Asia J. Public Health 2013, 2, $13-17$. [CrossRef]

6. NCD Risk Factor Collaboration (NCD-RisC). Worldwide trends in body-mass index, underweight, overweight, and obesity from 1975 to 2016: A pooled analysis of 2416 population-based measurement studies in 128.9 million children, adolescents, and adults. Lancet 2017, 390, 2627-2642. [CrossRef]

7. National Institute of Nutrition (Viện Dinh Dưỡng). Data on Children Malnutrition Annually (in Vietnamese). Available online: http:/ /viendinhduong.vn/news/vi/106/61/a/so-lieu-thong-ke-ve-tinh-trang-dinhduong-tre-em-qua-cac-nam.aspx (accessed on 14 March 2018).

8. United Nations Children's Funds, World Health Organization, World Bank. Joint Child Malnutrition Estimates 2017 (UNICEF-WHO-WB). Available online: http://apps.who.int/gho/data/node.wrapper. nutrition-2016?lang=en (accessed on 20 March 2018).

9. Jitnarin, N.; Kosulwat, V.; Rojroongwasinkul, N.; Boonpraderm, A.; Haddock, C.K.; Poston, W.S.C. Prevalence of overweight and obesity in Thai population: Results of the National Thai Food Consumption Survey. Eat Weight Disord. 2011, 16, e242-e249. [CrossRef] [PubMed]

10. Rerksuppaphol, S.; Rerksuppaphol, L. Prevalence of overweight and obesity among school children in suburb Thailand defined by the international obesity task force standard. J. Med. Assoc. Thai. 2010, 93, 27-31.

11. Naidu, B.M.; Mahmud, S.Z.; Ambak, R.; Sallehuddin, S.M.; Mutalip, H.A.; Saari, R.; Sahril, N.; Hamid, H.A.A. Overweight among primary school-age children in Malaysia. Asia Pac. J. Clin. Nutr. 2013, 22, 408-415. [PubMed]

12. De Onis, M.; Dewey, K.G.; Borghi, E.; Onyango, A.W.; Blössner, M.; Daelmans, B.; Piwoz, E.; Branca, F. The World Health Organization's global target for reducing childhood stunting by 2025: rationale and proposed actions. Matern. Child Nutr. 2013, 9, 6-26. [CrossRef] [PubMed]

13. Dieu, H.T.T.; Dibley, M.J.; Sibbritt, D.; Hanh, T.T.M. Prevalence of overweight and obesity in preschool children and associated socio-demographic factors in Ho Chi Minh City, Vietnam. Int. J. Pediatr. Obes. 2007, 2, 40-50. [CrossRef] [PubMed]

14. Nguyen, B.K.L.; Hop, L.T.; Do, V.A.N.; Tran, T.N.; Nguyen, H.C.; Tran, T.D.; Deurenberg, P.; Khouw, I. Double burden of undernutrition and overnutrition in Vietnam in 2011: Results of the SEANUTS study in 0.5-11-year-old children. Br. J. Nutr. 2013, 110, S45-S56. [CrossRef] [PubMed]

15. Fishman, S.; Caufield, L.E.; de Onis, M.; Blössner, M.; Hyder, A.A.; Mullany, L.; Black, R.E. Childhood and maternal underweight. Comparative Quantification of Health Risks: Global and Regional Burden of Disease Attributable to Selected Major Risk Factors; World Health Organization: Geneva, Switzerland, 2004.

16. Müller, O.; Krawinkel, M. Malnutrition and health in developing countries. CMAJ 2005, 173, $279-286$. [CrossRef] [PubMed]

17. World Health Organization. Obesity and Overweight. Available online: http://www.who.int/mediacentre/ factsheets/fs311/en/ (accessed on 18 March 2018).

18. Khan, N.C.; Khoi, H.H. Double burden of malnutrition: The Vietnamese perspective. Asia Pac. J. Clin. Nutr. 2008, 17, 116-118. [PubMed] 
19. Centers for Disease Control and Prevention. National Health and Nutrition Examination Survey (NHANES): Anthropometry Procedures Manual. Available online: https://www.cdc.gov/nchs/data/nhanes/nhanes_ 07_08/manual_an.pdf (accessed on 30 April 2016).

20. World Health Organization. WHO Anthro (Version 3.2.2, January 2011) and Macros: Software for Assessing Growth and Development of the World's Children. Available online: http:/ / www.who.int/childgrowth/ software/en/ (accessed on 19 October 2016).

21. World Health Organization. BMI-for-Age (5-19 years). Available online: http:/ / www.who.int/growthref/ who2007_bmi_for_age/en/ (accessed on 14 June 2012).

22. Nandy, S.; Irving, M.; Gordon, D.; Subramanian, S.V.; Smith, G.D. Poverty, child undernutrition and morbidity: New evidence from India. Bull. World Health Organ. 2005, 83, 210-216. [PubMed]

23. Nambiar, S.; Hughes, I.; Davies, P.S. Developing waist-to-height ratio cut-offs to define overweight and obesity in children and adolescents. Public Health Nutr. 2010, 13, 1566-1574. [CrossRef] [PubMed]

24. General Statistics Office of Vietnam. Average Income per Month Based on the Current Market by Rural/Urban Areas and Regions (in Vietnamese). Available online: http:/ /www.gso.gov.vn/SLTK/Menu. aspx?rxid=0ead611b-3d28-40ec-aed2-f5fc61041fe2\&px_language=vi\&px_db=11.+Y+toE1\%BA \%BF\%2c+ $\mathrm{v} \% \mathrm{C} 4 \% 83 \mathrm{n}+\mathrm{h} \% \mathrm{C} 3 \% \mathrm{~B} 3 \mathrm{a}+\mathrm{v} \% \mathrm{C} 3 \% \mathrm{~A} 0+\% \mathrm{C} 4 \% 91 \% \mathrm{E} 1 \% \mathrm{BB} \% 9 \mathrm{Di}+\mathrm{s} \% \mathrm{E} 1 \% \mathrm{BB} \% 91 \mathrm{ng} \& \mathrm{px}$-type=PX (accessed on 23 March 2016).

25. Hoang, T.D.N.; Le, D.T.; Pham, V.P.; Nambiar, S. Childhood overweight and obesity amongst primary school children in Hai Phong City, Vietnam. Asia Pac. J. Clin. Nutr. 2018, 27, 399-405.

26. Le, T.H.; Hoang, T.D.N. Prevalence of Overweight and Obesity and Associated Factors among Primary School Children in Hai Phong City in 2012 (in Vietnamese; National Institute of Nutrition Research: Hanoi, Vietnam, 2012.

27. Vietnam Ministry of Health. School Meal Programme for Primary School Children Is Going to Be Managed through a Software (in Vietnamese). Available online: http://moh.gov.vn/news/Pages/ ChuongTrinhMucTieuQuocGiaYTe.aspx?ItemID=2496 (accessed on 26 August 2017).

28. General Department of Preventive Medicine. Methods to Prevent Childhood Overweight, Obesity Period 2016-2020 (in Vietnamese). Available online: http:/ /vncdc.gov.vn/vi/phong-chong-benh-khong-lay-nhiem/ 744/giai-phap-phong-chong-thua-can-beo-phi-tre-em-giai-doan-2016-2020 (accessed on 6 April 2018).

29. People Committee of Hai Phong City. Competitive Advantage (in Vietnamese). Available online: http:/ /hppc.gov.vn/151082-110/10521510-n132.htm (accessed on 28 March 2018).

30. National Institute of Nutrition (Viện Dinh Dưỡng). Prevention and Treatment for Overweight/Obesity (in Vietnamese). Available online: http://viendinhduong.vn/chitietbancobietdinhduongvaphattrien.aspx? $\mathrm{id}=231$ (accessed on 3 April 2013).

31. National Institute of Nutrition-United Nations Children's Fund. National Nutrition Survey 2009-2010 (in Vietnamese). Available online: http:/ /viendinhduong.vn/FileUpload/Documents/010711_044347tinh_ hinh_dinh_duong_viet_nam_2009-2010.pdf (accessed on 20 April 2016).

32. Pham, Q.T.; Worsley, A. Middle-class household food providers' views and experiences of food marketing in Vietnam. Asia Pac. J. Clin. Nutr. 2016, 25, 863-870. [PubMed]

33. Swinburn, B. Obesity prevention in children and adolescents. Child Adolesc. Psychiatr. Clin. N. Am. 2009, 18, 209-223. [CrossRef] [PubMed]

34. Ueda, M.H.; Porto, R.B.; Vasconcelos, L.A. Food advertising and children's food choices. Psic.: Teor. e Pesq. 2014, 30, 53-61.

35. Nestle, M. Food marketing and childhood obesity-a matter of policy. N. Engl. J. Med. 2006, 354, $2527-2529$. [CrossRef] [PubMed]

36. Phan, T.B.N.; Dinh, T.H.; Hoang, T.S.; Pham, V.L. Assess the nutrition status of primary school children in Hue City (in Vietnamese). J. Pract. Med. 2009, 4, 46-50.

37. Corsi, D.J.; Mejía-Guevara, I.; Subramanian, S.V. Risk factors for chronic undernutrition among children in India: Estimating relative importance, population attributable risk and fractions. Soc. Sci. Med. 2016, 157, 165-185. [CrossRef] [PubMed]

38. Agbozo, F.; Atito, P.; Abubakari, A. Malnutrition and associated factors in children: A comparative study between public and private schools in Hohoe Municipality, Ghana. BMC Nutr. 2016, 2. [CrossRef]

39. Hoang, T.D.N.; Le, D.T.; Cao, T.T.H.; Nguyen, T.K.T. The prevalence of malnutrition among children under 5 years old was high in two communes in Ninh Thuan province, results from a survey conducted in 2017 (in Vietnamese). J. Food Nutr. Sci. 2017, 13, 32-39. 
40. Hoang, T.D.N.; Hop, L.T.; Huong, V.D.; Huong, C.T.H. The association between diet/physical activity and childhood overweight/obesity among primary school children and impacts of households socio-economic status (in Vietnamese). J. Food Nutr. Sci. 2014, 10, 7-13.

41. Tran, T.M.H.; Tran, T.H.L.; Pham, N.O.; Nguyen, N.T.; Le, K.H.; Ho Chi Minh Nutrition Centre. Nutrition status of school children in Ho Chi Minh City between 2002 and 2004 (in Vietnamese). Personal communication, 2005.

42. Tran, T.H.L.; Nguyen, T.K.H. Overweight and obesity in the population in Ho Chi Minh City (in Vietnamese). J. Food Nutr. Sci. 2005, 1, 1.

43. Lindsay, A.C.; Sussner, K.M.; Kim, J.; Gortmaker, S. The role of parents in preventing childhood obesity. Future Child. 2006, 16, 169-186. [CrossRef] [PubMed]

44. Belanger, D. Son preference in a rural village in North Vietnam. Stud. Fam. Plann. 2002, 33, 321-334. [CrossRef] [PubMed]

45. McCrory, M.A.; Fuss, P.J.; McCallum, J.E.; Yao, M.; Vinken, A.G.; Hays, N.P.; Roberts, S.B. Dietary variety within food groups: Association with energy intake and body fatness in men and women. Am. J. Clin. Nutr. 1999, 69, 440-447. [CrossRef] [PubMed]

46. Van Lierop, A.; Nam, N.V.; Doak, C.; Hung, L.Q.; Binh, T.Q.; Hoekstra, J.; de Vries, P.J. Regional clustering of anthropometric dimensions of primary school children in rural and suburban Vietnam. Asia Pac. J. Clin. Nutr. 2008, 17, 603-607. [PubMed]

47. Zhou, D.; Yang, M.; Yuan, Z.P.; Zhang, D.D.; Liang, L.; Wang, C.L.; Zhang, C.; Zhu, H.-H.; Lai, M.-D.; Zhu, Y.-M.; et al. Waist-to-height ratio: A simple, effective and practical screening tool for childhood obesity and metabolic syndrome. Prev. Med. 2014, 67, 35-40. [CrossRef] [PubMed]

48. Rerksuppaphol, S.; Rerksuppaphol, L. Waist circumference, waist-to-height ratio and body mass index of Thai children: Secular changes and updated reference standards. J. Clin. Diagn. Res. 2014, 8, PC05-PC09. [CrossRef] [PubMed]

(C) 2018 by the authors. Licensee MDPI, Basel, Switzerland. This article is an open access article distributed under the terms and conditions of the Creative Commons Attribution (CC BY) license (http:/ / creativecommons.org/licenses/by/4.0/). 